Draft: 18 Mar. 2006

\title{
Molecular outflows around high-mass young stellar objects
}

\author{
Y. Xu ${ }^{1,2}$, Z. Q. Shen ${ }^{1}$, J. Yang ${ }^{3}$, X. W. Zheng ${ }^{4}$, A. Miyazaki ${ }^{1}$, K. Sunada ${ }^{5}$, H. J. Ma ${ }^{3}$, J. J. \\ $\mathrm{Li}^{1,6}$, J. X. Sun ${ }^{3}$ and C. C. Pei ${ }^{3}$
}

\begin{abstract}
We present a study of molecular outflows using high-resolution mapping of the $\mathrm{CO}$ (1-0) line emission toward eight relatively nearby $6.7 \mathrm{GHz}$ methanol masers, which are associated with massive star forming regions. Outflows were detected in seven out of eight sources, and five of them clearly show bipolar or multiple outflow morphologies. These outflows have typical masses of a few solar masses, momenta of tens of $M_{\odot} \mathrm{km} \mathrm{s}^{-1}$, kinetic energies of $\sim 10^{45} \mathrm{ergs}$, and the mass entrainment rate of a few $10^{-5} M_{\odot} \mathrm{yr}^{-1}$. They have significantly more mass and kinetic energy than their low-mass counterparts. In some of the sources, the massive outflow is obviously associated with a particular massive star in the cluster, while in others the origin remains uncertain. The high detection rate of outflows toward methanol masers suggests that the outflow phase of massive protostars encompasses the methanol maser phase.
\end{abstract}

Subject headings: stars: formation- ISM: jets and outflows -ISM: clouds

\section{INTRODUCTION}

Molecular outflows may be the main signature of the earliest stage in massive star formation. Outflows have been investigated with CO molecule lines by many authors (Bally

\footnotetext{
${ }^{1}$ Shanghai Astronomical Observatory, Chinese Academy of Sciences, Shanghai 20030, China; yxu@shao.ac.cn

${ }^{2}$ Max-Planck-Institut für Radioastronomie, Auf dem Hügel 69, 53121 Bonn, Germany; xuye@mpifrbonn.mpg.de

${ }^{3}$ Purple Mountain Observatory, Nanjing, 210008, China

${ }^{4}$ Department of Astronomy, Nanjing University Nanjing 210093, China

${ }^{5}$ Nobeyama Radio Observatory, Nagano, 384-13, Japan

${ }^{6}$ Graduate School of Chinese Academy of Sciences, Beijing 100039, China
} 
\& Lada 1983; Lada 1985; Snell et al. 1988; 1990), since Zuckerman et al. (1976) and Kwan \& Scoville (1976) discovered them. However, most studies are toward low-mass star-forming regions. Recently some systematic observations toward massive star-forming regions have been carried out (Shepherd \& Churchwell 1996; Zhang et al. 2001; 2005; Beuther et al. 2002). It is difficult to determine the characteristics of outflows associated with massive YSOs because there are relatively fewer massive stars, they tend to form in clusters, and they are often at distances greater than $1 \mathrm{kpc}$. Therefore, a moderate resolution observation is critical to achieve the necessary degree of kinematic and morphological details. Because of this, most of the observations were carried out with the ${ }^{12} \mathrm{CO}(2-1)$ line, while only a few sources were studied by using the ${ }^{12} \mathrm{CO}$ (1-0) line with a high resolution. The excitation energy of the $\mathrm{J}=2$ level is $16 \mathrm{~K}$ above the ground state, while it is only $5 \mathrm{~K}$ for the $\mathrm{J}=1$ level. Thus the $\mathrm{J}=2$ level (and higher) can better represent the conditions thought to be in outflows, while the $\mathrm{J}=1$ level can trace the cooler, non-star-forming gas in the galactic plane. Obviously, having two different transitions observed will be helpful to our better understanding of the physical properties of outflows.

In order to investigate the structure and dynamics of outflows associated with massive star-forming regions on a finer scale, we performed ${ }^{12} \mathrm{CO}$ (1-0) observations of $6.7 \mathrm{GHz}$ methanol masers in a sample of massive star-forming regions (Xu et al. 2003) with the Nobeyama 45-m telescope in Japan. It is the first systematic study with a high resolution observation $\left(\sim 15^{\prime \prime}\right)$ of the $\mathrm{CO}(1-0)$ line. The $6.7 \mathrm{GHz}$ methanol maser (a Class II methanol maser transition) is often found to be associated with massive star forming regions (Menten 1991; Walsh et al. 1998; van der Walt et al. 2003). The selecting criteria are (1) declination $\delta \geq-10^{\circ}$; and $(2)$ distance $\mathrm{d} \leq 1.5 \mathrm{kpc}$. There are eight sources that satisfy these criteria (Table 1). These sources have infrared luminosities between about $10^{3} L_{\odot}$ and $10^{4} L_{\odot}$. Most of them have been observed previously in CO by other single dish telescopes. Both IRAS 06053-0622 and 22543+6145 were also observed by interferometer (Minier et al. 2000). Our observations have a linear resolution of better than $\sim 0.1 \mathrm{pc}$, about one tenth of the typical length of an outflow. Thus they should be able to well determine the morphology of outflows.

\section{OBSERVATIONS}

The observations were made in 2003 April at the ${ }^{12} \mathrm{CO} J=1-0$ emission line $(115.271202 \mathrm{GHz})$ with the 45-m radio telescope of the Nobeyama Radio Observatory (NRO) in Japan. The names, positions, and velocities of the observed 8 IRAS sources are summarized in Table 1. We used the 25-beam array receiver system (BEARS), which is the $5 \times 5$ focal-plane array SIS mixer receiver working in the 80-115 GHz band (Sunada et al. 2000). Typical main 
beam efficiency and half power beam width (HPBW) for each beam at $115 \mathrm{GHz}$ are 0.45 and $15^{\prime \prime}$, respectively. The pointing accuracy was about $5^{\prime \prime}$ and was checked by observing nearby $\mathrm{SiO}$ maser sources at $43 \mathrm{GHz}$ every one hour. Since the beam separation on the sky is $\sim 41^{\prime \prime}$, we observed the interpolated two points between each beam using the multi ON-OFF position switching technique, and then made the final map with the grid spacing of $\sim 14^{\prime \prime}$ in an area of $3^{\prime} \times 3^{\prime}$ around the IRAS sources. The OFF positions are about $1^{\circ}$ away from each source. The typical system temperature during the observations was $T_{\text {sys }}(\mathrm{DSB})$ $=450 \mathrm{~K}$. The intensity scale of the spectra was calibrated by the chopper wheel method. The spectrometer is an auto correlator of high resolution mode with a $32 \mathrm{MHz}$ bandwidth and a $37.8 \mathrm{kHz}$ spectral resolution, corresponding to a velocity resolution of $\sim 0.1 \mathrm{~km} \mathrm{~s}^{-1}$ at $115 \mathrm{GHz}$.

The intensities of the spectra were corrected using the scaling factor provided by the NRO to calibrate the the sideband rejection ratio of each BEARS element and to establish the absolute intensity scale. The scaling factors of each beam are estimated by comparing the peak intensities in $\mathrm{S} 140$ in the ${ }^{12} \mathrm{CO} J=1-0$ line with those measured with a single sideband SIS receiver (S100). Then the line intensity is reported in terms of the corrected antenna temperature $T_{\mathrm{R}}^{*}$. The main beam efficiency with $\mathrm{S} 100$ at $115 \mathrm{GHz}$ is 0.40 . The total integration time of each position was about 6 minutes, which achieved a RMS noise level of $\sim 0.2 \mathrm{~K}\left(T_{\mathrm{A}}^{*}\right)$ at the velocity resolution of $\sim 0.1 \mathrm{~km} \mathrm{~s}^{-1}$. The data were reduced by using the NEWSTAR reduction package of the NRO. After subtracting linear baselines, the data were smoothed to a resolution of $\sim 0.5 \mathrm{~km} \mathrm{~s}^{-1}$ to improve the signal to noise ratio.

\section{RESULTS AND DISCUSSION}

Molecular outflows were detected in all sources, except IRAS 22551+6139. The mapping results of the integrated CO line wing emission are shown in Figure 1. Five sources (IRAS 00338+6312, IRAS 06053-0622, IRAS 06056+2131, IRAS 20081+3122 and IRAS $22543+6145$ ) show a clear bipolar or multiple outflow structure. Except in IRAS 06053-0622, the methanol masers are located near the center of the bipolar outflows. One source (IRAS $22272+6358$ ) appears to have a one-sided lobe. The ranges of red and blue wing emission, the integrated flux at the position of maximum integrated intensity, and the collimation factor (Lada 1985) are listed in Table 2.

The outflow parameters, except for $\mathrm{CO}$ column density $N_{C O}$ which is derived from Snell et al. (1988), are estimated with the method of Beuther et al. (2002). We assume that the gas is in local thermodynamic equilibrium at a gas temperature of $30 \mathrm{~K}$, and a $\mathrm{CO}$ to $\mathrm{H}_{2}$ abundance ratio of $10^{-4}$ (Snell et al. 1988). The physical properties of outflows 
are summarized in Table 3. We here are using the optically-thin assumption, and masses and energetics in Table 3 are lower-limits to the true values. These are about an order of magnitude less than the values with the method of Beuther et al. (2002), where they corrected for optical depth effects in their data. Typically, the molecular outflows have masses of a few solar masses, momenta of tens of $M_{\odot} \mathrm{km} \mathrm{s}^{-1}$, kinetic energies of $\sim 10^{45}$ ergs, mass entrainment rate of a few $10^{-5} M_{\odot} \mathrm{yr}^{-1}$, mechanical force of a few $10^{-4} M_{\odot} \mathrm{km}$ $\mathrm{s}^{-1} \mathrm{yr}^{-1}$, and mechanical luminosity of about $1 L_{\odot}$, respectively. These results show that outflows from these massive star forming regions have significantly more mass and kinetic energy than those from low-mass star forming regions (Myers et al. 1988, Cabrit \& Bertout 1992, Bontemps et al. 1996).

\subsection{Comments on Individual Sources}

IRAS 00338+6312 IRAS $00338+6312$ is located near the core of the dark cloud L1287. The methanol maser is coincident with the IRAS source. A bipolar outflow was first detected by Snell et al. (1990) and confirmed by Yang et al. (1991). The outflow is in the NE-SW direction and shows a clear bipolarity. Evans et al. (1994) mapped CO (3-2) with a comparable resolution of $20^{\prime \prime}$ to our CO (1-0) observations. They computed a range of masses for the outflow using the optically-thin assumption for the CO (3-2) data for the lower limit, and an optical-depth correction for the upper limit. The lower limit outflow mass derived by them is similar to that listed in Table 3 derived from the CO (1-0) data, as we would expect given the optically-thin assumption for them. The IRAS source lies midway between the center of the blue and red lobes. There is the FU Ori star RNO1B located about 11" south of the IRAS peak (Evans et al. 1994). However, the CO outflow appears to be driven not by RNO1B, but by a source at or closer to the IRAS source. A radio continuum source with a positive spectral index (Anglada et al. 1994), and a core observed with CS, HCN, $\mathrm{HCO}^{+}$ and $\mathrm{NH}_{3}$ (Yang et al. 1991; Walker \& Masheder 1997; Zinchenko et al. 1997) are coincident with the IRAS source. All of these tracers appear to originate from the same source that is driving the bipolar outflow.

IRAS 06053-0622 There is a large offset $\left(18^{\prime \prime}, 3^{\prime \prime}\right)$ between the methanol maser and IRAS 06053-0622 which is slightly off a compact HII region ( $7^{\prime \prime}, 8^{\prime \prime}$, Walsh et al. 1998). The outflow in this source has been widely studied by many authors. With a resolution of about 120", Bally and Lada (1983) firstly detected a large bipolar outflow with a maximal radius of up to 2 pc. Observation with a higher resolution $\left(60^{\prime \prime}\right)$ shows that the outflow consists of a blue wing and two separated red lobes (Meyers-Rice \& Lada 1991). Our observations with a 
better resolution of $15^{\prime \prime}$ reveal that the outflow roughly consists of two pairs of bipolar lobes. The emission from the red lobe is stronger than that from the blue lobe. The less-dominant one with poor collimation factor is situated to the north. There are a lot of infrared sources in this region (Aspin \& Walther 1990; Yao et al. 1997). IRAS 06053-0622 is surrounded by IRS 1, 2, 3 and $\mathrm{a}_{i}$. The methanol maser coincides with $\mathrm{CS}, \mathrm{H}_{2} \mathrm{CO}, \mathrm{HCN}$ and $\mathrm{H}_{2} \mathrm{CO}$ peaks which are associated with infrared source IRS 3 (Giannakopoulou et al. 1997; Choi et al. 2000). Since the IRAS source, IRS 3 and the compact HII region are far away from the center of the dominant outflow, they are not likely to be the exciting source of the outflow. IRS 6 lies midway between peaks of blue and red lobes of the dominant one, and so probably it is this infrared source that drives the outflow. No infrared source is near the less-dominant outflow.

IRAS 06055+2039 Although no outflow was claimed previously, broad CO wing emission was seen by Shepherd \& Churchwell. (1996). Our image shows an outflow. Although the outflow appears in both red and blue wings, they seem not to be a bipolar outflow. The two lobes have different orientations. The red one extends in the SW-NE direction, while the blue one is almost perpendicular to the red one. There is a large offset $\left(40^{\prime \prime}, 17^{\prime \prime}\right)$ between the methanol maser and the IRAS source. The center of red wing is closer the methanol $\left(15^{\prime \prime}, 0\right)$ than the IRAS source $\left(30^{\prime \prime}, 15^{\prime \prime}\right)$. The methanol maser is coincident with a compact HII region, an $\mathrm{H}_{2} \mathrm{O}$ maser, and $\mathrm{CS}$ and far-infrared peaks (Lada et al. 1981; Shepherd \& Churchwell 1996; Zinchenko et al. 1998; Tej et al. 2006). Therefore, they are the same source as the exciting source of the red wing. The center of the blue wing coincides with a far-infrared core (Tej et al. 2006), indicating a possible exciting source of the outflow.

IRAS $06056+2131$ IRAS $06056+2131$ is located in a complex of molecular clouds. The methanol maser is nearly coincident with the IRAS source and an UC HII region (Kurtz et al. 1994). Snell et al. (1988) detected a CO bipolar outflow, which is in the northeastsouthwest direction, and peaks of blue and red wings are spatially separated. From figure 1 there are two bipolar outflows, one (outflow 1 detected by Snell et al. 1988) on the upper-left and another, outflow 2, newly detected by Xu et al. (2004) on the right. Zhang et al. (2005) obtained a similar result with a resolution of about $30^{\prime \prime}$. The IRAS source is associated with outflow 2. The blue-shifted emission is stronger than the red one in outflow 1 while in contrast the red-shifted lobe is dominant in outflow 2. Since the mapping area is limited, the blue lobe of outflow 1 is not shown entirely. From low resolution mapping of Snell et al. (1988), it should stretch beyond the map boundary to the east. The red wing of outflow 2 extends from the west to the east and connects outflow 2 to outflow 1 . Therefore, outflow 1 can also be regarded as a multiple outflow since its blue wing connects the red wings of the 
two outflows. There are a lot of objects in this cloud. Near-infrared polarimetric images show that there are two infrared nebulae correspondingly associated with the two outflows (Yao et al. 2000). The nebula near the outflow 2 is associated with a cluster of stars. Outflow 2 is associated with a water source, ammonia and CS emission peaks, and an UC HII (Verdes et al. 1989; Kompe et al. 1989; Kurtz et al. 1994; Morata et al. 1997). These indicate that there are at least a massive star and a cluster of low- and intermediate mass stars associated with outflow 2. Due to its large momentum, outflow 2 is most likely to be driven by the UC HII region. Outflow 1 is associated with an infrared nebula and a CS peak. This outflow may be driven by an embedded massive star/stars associated with the nearby infrared nebula.

IRAS 20081+3122 Yang et al. (2002) detected a high-velocity CO line wing in this region. Kumar et al. (2004) detected a bipolar outflow using $\mathrm{H}^{13} \mathrm{CO}^{+}$line. Our observations of the $\mathrm{CO}$ line also show a bipolar outflow, though the orientation is slightly different. The red lobe extends roughly along the EW direction, and the blue one along the NS direction, while the $\mathrm{H}^{13} \mathrm{CO}^{+}$outflow aligns in the SW-NE direction. The length of the former is nearly as two times as the latter. The methanol maser is associated with IRAS $20081+3122, \mathrm{NH}_{3}$ and mm continuum peaks and other masers, such as $44 \mathrm{GHz}$ methanol, $22 \mathrm{GHz} \mathrm{H}_{2} \mathrm{O}$ and $6 \mathrm{GHz} \mathrm{OH}$ masers (Zheng et al. 1985; Desmurs \& Baudry 1998; Kurtz et al. 2004; Kumar et al. 2004; Kurtz \& Hofner 2005). Previous observations of $\mathrm{cm}$ continuum emission reveal an UC HII region which coincides with the IRAS source (Turner \& Matthews 1984; Zheng et al. 1985; Kurtz et al. 2004) and is almost located at the center of the outflow, indicating a possible exciting source of the outflow.

IRAS 22272+6358 An outflow was only detected in blue lobe, as firstly discovered by Sugitani et al. (1989). The methanol maser is coincident with IRAS $22272+6358$ and a $\mathrm{NH}_{3}$ core (Zinchenko et al. 1997). Because no cm continuum emission has been detected, this source may be too young to have produced a detectable compact HII region (Wilking et al. 1989). Near-infrared observation shows that this source is a heavily extinguished object (Ressler \& Shure 1991). The IRAS source is close to the center of the outflow, indicating a possible exciting source of the outflow.

IRAS 22543+6145 Though outflows in this region have been investigated by many authors (Bally \& Lada 1983; Richardson et al. 1987; Narayanan \& Walker 1996), our observation is the first single dish study at a resolution of $15^{\prime \prime}$. Figure 1 clearly shows a bipolar structure with a relatively poor collimation. The CO (3-2) maps show a similar configuration to our result (Narayanan \& Walker 1996). We could not confirm it due to the finite mapping area. 
The methanol maser is coincident with IRAS $22543+6145$, a mm emission peak and a CS core, and near the edge of an $\mathrm{NH}_{3}$ core (Torrelles et al. 1993; Narayanan \& Walker 1996). The IRAS source lies midway between two compact HII regions (Hughes \& Wouterloot 1984; Garay et al. 1996). The separation $\left(9^{\prime \prime}, 2^{\prime \prime}\right)$ between the IRAS source and the two HII regions is less than the IRAS error ellipse $\left(10^{\prime \prime}, 5^{\prime \prime}\right)$. We could not determine whether the IRAS source is associated with either HII region. Since they all reside near the center of the outflow, it is unclear which source drives the molecular outflow. The IRAS source lies midway between the center of both blue and red lobes, indicating that it is most likely to denote the location of driving source of the outflow. There seems to be a second red lobe at the South, which is likely to be the red wing of another outflow, but it is uncertain due to the limited mapping area.

IRAS 22551+6139 No outflow was detected in this region. Although there is a large infrared luminosity, non-detection of radio continuum indicates that this source is associated with an earlier stage of the star formation process, before the embedded star has had a chance to form an UC compact HII region. At the time, an outflow may be too small or/and weak to detect.

\subsection{DISCUSSION}

It is believed that massive outflows occur at the early phases of protostellar evolution and disappear when a compaction HII region evolves sufficiently. Thus, the exciting source is either a massive protostar or an UC/compact HII region. There is evidence that methanol masers may trace a more narrowly constrained stage in the life of a massive protostar. They appear to arise in hot molecular cores but disappear sometime before the HII region phase (Minier et al. 2002). The outflows were detected in seven out of eight sources. Five methanol masers are associated with compact HII regions and located near the center of the outflows, indicating that they could denote the location of the driving sources of these outflows. However, not all of the compact HII regions are the exciting sources of the outflows. In IRAS 06053-0622 and IRAS 06056+2131 (outflow 1), obviously the exciting sources are not the known compact HII regions. In IRAS $22543+6145$, it is unclear which source is the exciting source of the outflow, maybe one of the two compact HII regions or the IRAS source. There is no compact HII region at all in IRAS $22272+6358$ and so the exciting source is probably a massive protostar. Since massive stars are usually born in clusters, massive outflows are most likely to be excited by one of massive stars in a cluster, such as IRAS $22543+6145$. On the other hand, from Table 3 the dynamical timescale of the outflows is 
about a few $10^{4} \mathrm{yr}$, while an UC HII regions could live a few $10^{5}$ yr (Churchwell 1999). In our sample, outflows were detected in seven out of eight sources. The high detection rate of outflows toward methanol masers suggests that the duration of the outflow phase encompasses the methanol maser phase.

\section{SUMMARY}

We mapped the CO (1-0) emission toward eight massive star forming regions using the 45-m radio telescope of the $\mathrm{NRO}$ in Japan. With one exception all sources were detected to be associated with massive outflows. Two are newly detected in the CO (1-0) line, and some show much finer structures than before. Our observations have detected an outflow in IRAS $06055+2039$ for the first time; The outflow configuration detected in the CO (10 ) line is slightly different from that in the $\mathrm{H}^{13} \mathrm{CO}^{+}$line in IRAS 20081+3122; Multiple outflows are definitely detected in IRAS 06053-0622 and IRAS 06056+2131, and most likely in IRAS $22543+6145$ as well. These outflows have significantly more mass and kinetic energy than their low-mass counterparts. Some massive outflows are obviously associated with a particular massive star in the cluster. This is consistent with the high detection rate but the short dynamical timescale of the outflows.

We are grateful to the NRO staff for the excellent support. We thank the anonymous referee for many useful suggestions and comments, which greatly improved this paper. We thank K. Tatematsu for the help of the data reduction. This work is supported in part by the

National Science Foundation of China under grants 10133020, 10373025 and 10573029. Z.-Q. Shen acknowledges support by the One-Hundred-Talent Program of the Chinese Academy of Sciences.

\section{REFERENCES}

Anglada, G., Rodriguez, L. F., Girart, J. M., et al. 1994, ApJ, 420, L91

Aspin, C., \& Walther, D. M. 1990, A\&A, 235, 387

Bally, J., \& Lada, C. J. 1983, ApJ, 265, 824

Beuther, H., Schilke, P., Sridharan, T.K. et al. 2002, A\&A, 383, 892

Bontemps, S., André, P., Terebey, S., \& Cabrit, S. 1996, A\&A, 311, 858

Cabrit, S., \& Bertout, C. 1992, A\&A, 261, 274 
Casoli, F., Dupraz, C., Gerin, M., Combes, F., \& Boulanger, F. 1986, A\&A, 169, 281

Churchwell, E, 1999, The Origin of Stars and Planetary Systems. Edited by Charles J. Lada and Nikolaos D. Kylafis. Kluwer Academic Publishers, 1999, p. 515

Choi, M., Evans II, N. J., Tafalla, M., \& Bachiller, R. 2000, ApJ, 538, 738

Desmurs, J. E., \& Baudry, A. 1998, A\&A, 340, 521

Evans, N. J., Stephen, B., Russell, M. L., Lee, H., \& Scott, K. 1994, ApJ, 424, 973

Garay, G., Ramirez, S., Rodriguez, Luis F. et al. 1996, ApJ, 496, 193

Giannakopoulou, J., Mitchell, G. F., Hasegawa, T. I. et al. 1997, ApJ, 487, 346

Hughes V. A., \& Wouterloot J. G. A. 1984, ApJ, 276, 204

Kompe, C., Baudry, A., Joncas, G., \& Wouterloot, J. G. A. 1989 A\&A, 221, 295

Kumar, M. S. N., Tafalla, M., \& Bachiller, R. 2004, A\&A, 426, 195

Kurtz, S., Churchwell, E., \& Wood, D. O. S. 1994, ApJS, 91, 659

Kurtz, S., Hofner, P., \& Álvarez, C. V. 2004, ApJS, 155, 149

Kurtz, S., \& Hofner, P. 2005, AJ, 130, 711

Kwan, J., \& Scoville, N. 1976, ApJ, 210, L39

Lada, C. J. 1985, ARA\&A, 23, 267

Lada, C. J., Blitz, L., Reid, M. J., \& Moran, J. M. 1981, ApJ, 243, 769

Menten, K. 1991, ApJ, 380, L75

Meyers-Rice, B. A., \& Lada, C. J. 1991, ApJ, 368, 445

Morata, O., Estalella, R., Lopez, R., \& Planesas, P. et al 1997 MNRAS, 292, 120

Myers, P. C., Heyer, M., Snell, R. L., \& Goldsmith, P. F. 1988, ApJ, 324, 907

Minier, V., Booth, R. S., \& Conway, J. E., 2000, A\&A, 362, 1093

Minier, V., Booth, R. S., Burton, M. G., \& Pestalozzi, M. R., 2002, Proceedings of the 6th European VLBI Network Symposium Ros, E., Porcas, R.W., Lobanov, A.P., \& Zensus, J.A. (eds.) June 25th-28th 2002, Bonn, Germany, p. 205

Narayanan, G., \& Walker, C. K. 1996, ApJ, 466, 844 
Ressler, M. E., \& Shure, M. 1991, AJ, 102, 1398

Richardson, K. J., White, Glen J., Avery, L. W., \& Woodsworth, A. W. 1987, A\&A, 174, 197

Shepherd D.S., \& Churchwell, E. 1996, ApJ, 457,267

Snell, R. L., Huang, Y.-L., Dickman, R. L., \& Claussen, M. J. 1988, ApJ, 325, 853

Snell, R. L., Huang, Y.-L., Dickman, R. L., \& Claussen, M. J. 1990, ApJ, 352, 139

Sugitani, K., Fukui, Y., Mizuni, A., \& Ohashi, N. 1989, ApJ, 342, L87

Sunada, K., Yamaguchi, C., Nakai, N., et al. 2000, in Proc. SPIE Vol.4015, Radio Telescopes, ed. H.R. Butcher, 237

Tej, A., Ojha, D. K., Ghosh, S. K. et al. 2006, A\&A, in press

Torrelles, J. M., Verdes-Montenegro, L., \& Ho, P. T. P. 1993, ApJ, 410, 202

Turner, B. E., \& Matthews, H. E. 1984, ApJ, 277, 164

van der Walt, D. J., Churchwell, E., Gaylard, M. J., \& Goedhart, S. 2003, MNRAS, 341, 270

Verdes-Montenegro, L., Torrelles, J. M., Rodriguez, L. F. et al. 1989, ApJ, 346, 193

Walker, R. N. F., \& Masheder, M. R. X. 1997, MNRAS, 258, 862

Walsh, A. J., Burton, M. G., Hyland, A. R., \& Robinson, G. 1998, MNRAS, 301, 640

Wilking, B. A., Blackwell, J. H., Mundy, L. G., \& Howe, J. E., 1989, ApJ, 345, 257

Xu, Y., Zheng X. W., \& Jiang, D. R. 2003, ChJAA, 3, 1663

Xu, Y., Yang J., Zheng, X. W. et al. 2004, Chin. Phys. Letter., 21, 2071

Yang, J., Jiang, Z., Wang, M., Ju, B., \& Wang, H. 2002, ApJS, 141, 157

Yang, J., Umemoto, T., Iwata, T., \& Fukui, Y. 1991, ApJ, 373, 137

Yao, Y., Hirata, N., Ishii, M. et al. 1997, ApJ, 490, 281

Yao, Y., Ishii, M., Nagata, T. et al. 2000, ApJ, 542, 392

Zhang, Q., Hunter, T. R., Brand, J. et al. 2001, ApJ, 552, L167

Zhang, Q., Hunter, T. R., Brand, J. et al. 2005, ApJ, 625, 864

Zheng, X. W., Ho, P. T. P., Reid, M. J., \& Schneps, M. H. 1985, ApJ, 293, 522 
Zinchenko, I., Henning, Th., \& Schreyer, K. 1997, A\&AS, 124, 385

Zinchenko, I., Pirogov, L., \& Toriseva, M. 1998, A\&AS, 133, 337

Zuckerman, M, Kuiper, T. B. H., \& Rodriguez Kuiper, E. N. 1976, ApJ, 209, L137 
Table 1: List of Objects: Columns 1 and 2 are source names. Columns 3 and 4 list the positions of sources. $V_{L S R}$ (column 5) is the velocity of the line peak. Columns 6 and 7 are distances and infrared luminosities derived from IRAS flux densities (Casoli et al. 1986).

\begin{tabular}{|c|c|c|c|c|c|c|}
\hline IRAS & Name & $\begin{array}{l}\text { R.A.(2000) } \\
\left(\begin{array}{lll}\mathrm{h} & \mathrm{m} & \mathrm{s}\end{array}\right)\end{array}$ & 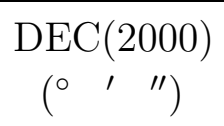 & $\begin{array}{c}V_{L S R} \\
\left(\mathrm{~km} \mathrm{~s}^{-1}\right)\end{array}$ & $\begin{array}{c}D \\
(\mathrm{kpc})\end{array}$ & $\begin{array}{l}L_{F I R} \\
\left(10^{3} L_{\odot}\right)\end{array}$ \\
\hline $00338+6312$ & L1287 & 003647.5 & 632902 & -18.3 & 0.9 & 0.8 \\
\hline $06053-0622$ & Mon R2 & 060748.0 & -062257 & 9.5 & 0.8 & 11.0 \\
\hline $06055+2039$ & $\mathrm{~S} 252 \mathrm{~A}$ & 060835.5 & 203859 & 8.6 & 1.5 & 6.0 \\
\hline $06056+2131$ & AFGL 6366S & 060841.2 & 213104 & 2.5 & 1.5 & 10.7 \\
\hline $20081+3122$ & $\mathrm{ON} 1$ & 201009.1 & 313137 & 11.1 & 1.4 & 7.5 \\
\hline $22272+6358$ & L1206 & 222852.2 & 641343 & -8.9 & 0.9 & 0.8 \\
\hline $22543+6145$ & Cep A East & 225619.1 & 620157 & -11.7 & 0.7 & 15.3 \\
\hline $22551+6139$ & & 225711.2 & 615603 & -10.7 & 0.6 & 7.8 \\
\hline
\end{tabular}

Table 2: Parameters of outflows: Columns $(2,3)$ and $(4,5)$ list the LSR velocity range $\Delta v$, and the peak integrated wing emission $S$ for the blue- and red-shifted wings, respectively. The last column is the collimation factor $f_{c} . \Delta v$ is measured at $T_{A}^{*}=0.2 \mathrm{~K}$ level $(2 \sigma)$ and included low-velocity outflows (Shepherd \& Churchwell 1996).

\begin{tabular}{cccccc}
\hline \hline $\begin{array}{c}\text { IRAS } \\
\text { Source }\end{array}$ & $\begin{array}{c}\Delta v_{b} \\
\left(\mathrm{~km} \mathrm{~s}^{-1}\right)\end{array}$ & $\begin{array}{c}\Delta v_{r} \\
\left(\mathrm{~km} \mathrm{~s}^{-1}\right)\end{array}$ & $\begin{array}{c}S_{b} \\
\left(\mathrm{~K} \mathrm{~km} \mathrm{~s}^{-1}\right)\end{array}$ & $\begin{array}{c}S_{r} \\
\left(\mathrm{~K} \mathrm{~km} \mathrm{~s}^{-1}\right)\end{array}$ & $f_{c}$ \\
\hline $00338+6312$ & $(-30,-19)$ & $(-14,-7)$ & 36.2 & 31.2 & 2.2 \\
$06053-0622 a$ & $(0,7)$ & $(15,23)$ & 22.4 & 28.0 & 1.7 \\
$06053-0622 b$ & $(0,7)$ & $(15,23)$ & 37.9 & 56.2 & 2.3 \\
$06055+2039$ & $(0,5.5)$ & $(12,17)$ & 29.6 & 36.1 & 3.2 \\
$06056+2131 a$ & $(-10,0)$ & $(5,15)$ & 24.7 & 12.4 & 1.7 \\
$06056+2131 b$ & $(-10,0)$ & $(5,15)$ & 17.0 & 25.6 & 1.8 \\
$20081+3122$ & $(-5,7.5)$ & $(15,20)$ & 17.5 & 17.9 & 2.7 \\
$22272+6358$ & $(-18,-11)$ & & 8.0 & & 2.6 \\
$22543+6145$ & $(-28,-14.5)$ & $(-6.5,6)$ & 35.8 & 28.3 & 1.6 \\
\hline
\end{tabular}


Table 3: Results of Outflows: Columns 2 and 3 give $\mathrm{H}_{2}$ column densities $N\left[10^{20} \mathrm{~cm}^{-2}\right]$ in both the blue- and red-shifted outflow lobes with the corresponding lobe masses in $M_{\odot}$ listed in columns 4 and 5 . Column 6 is the total mass $M_{\text {out }}\left[M_{\odot}\right]$. Columns 7 and 8 give the momentum $p\left[M_{\odot} \mathrm{km} \mathrm{s}^{-1}\right]$, and the energy $E\left[10^{45} \mathrm{erg}\right]$. The last five columns are the size $[\mathrm{pc}]$, the characteristic time scale $t\left[10^{4} \mathrm{yr}\right]$, the mass entrainment rate of the molecular outflow $\dot{M}_{\text {out }}\left[10^{-5} M_{\odot} \mathrm{yr}^{-1}\right]$, the mechanical force $F_{m}\left[10^{-4} M_{\odot} \mathrm{km} \mathrm{s}^{-1} \mathrm{yr}^{-1}\right]$, and the mechanical luminosity $L_{m}\left[L_{\odot}\right]$, respectively.

\begin{tabular}{ccccccccccccc}
\hline \hline $\begin{array}{c}\text { Source } \\
(1)\end{array}$ & $N_{b}$ & $N_{r}$ & $M_{b}$ & $M_{r}$ & $M_{\text {out }}$ & $p$ & $E$ & size & $t$ & $\dot{M}_{\text {out }}$ & $F_{m}$ & $L_{m}$ \\
$(2)$ & $(3)$ & $(4)$ & $(5)$ & $(6)$ & $(7)$ & $(8)$ & $(9)$ & $(10)$ & $(11)$ & $(12)$ & $(13)$ \\
\hline $00338+6312$ & 2.9 & 2.2 & 0.7 & 0.7 & 1.4 & 16.0 & 1.8 & 0.37 & 3.1 & 4.5 & 5.1 & 0.49 \\
$06053-0622 a$ & 2.6 & 3.1 & 0.4 & 0.6 & 1.0 & 11.0 & 1.4 & 0.26 & 2.2 & 4.5 & 5.3 & 0.53 \\
$06053-0622 b$ & 3.4 & 5.3 & 1.1 & 2.4 & 3.5 & 43.0 & 5.4 & 0.34 & 2.9 & 12.0 & 15.0 & 1.50 \\
$06055+2039$ & 2.8 & 3.3 & 3.3 & 3.4 & 6.7 & 60.0 & 5.5 & 0.80 & 8.6 & 7.7 & 6.9 & 0.51 \\
$06056+2131 a$ & 1.8 & 1.7 & 1.5 & 1.0 & 2.5 & 31.0 & 3.9 & 0.41 & 3.2 & 7.8 & 9.8 & 1.00 \\
$06056+2131 b$ & 1.6 & 2.5 & 0.9 & 2.2 & 3.1 & 39.0 & 4.9 & 0.68 & 5.3 & 6.0 & 7.5 & 0.77 \\
$20081+3122$ & 1.5 & 1.6 & 0.8 & 1.5 & 2.3 & 26.0 & 3.3 & 0.73 & 5.7 & 4.1 & 4.6 & 0.47 \\
$22272+6358$ & 0.5 & & 0.3 & & 0.3 & 2.5 & 0.2 & 0.49 & 5.2 & 0.5 & 0.5 & 0.04 \\
$22543+6145$ & 3.1 & 2.7 & 0.5 & 0.3 & 0.8 & 14.0 & 2.4 & 0.30 & 1.7 & 4.7 & 8.1 & 1.10 \\
\hline \hline
\end{tabular}



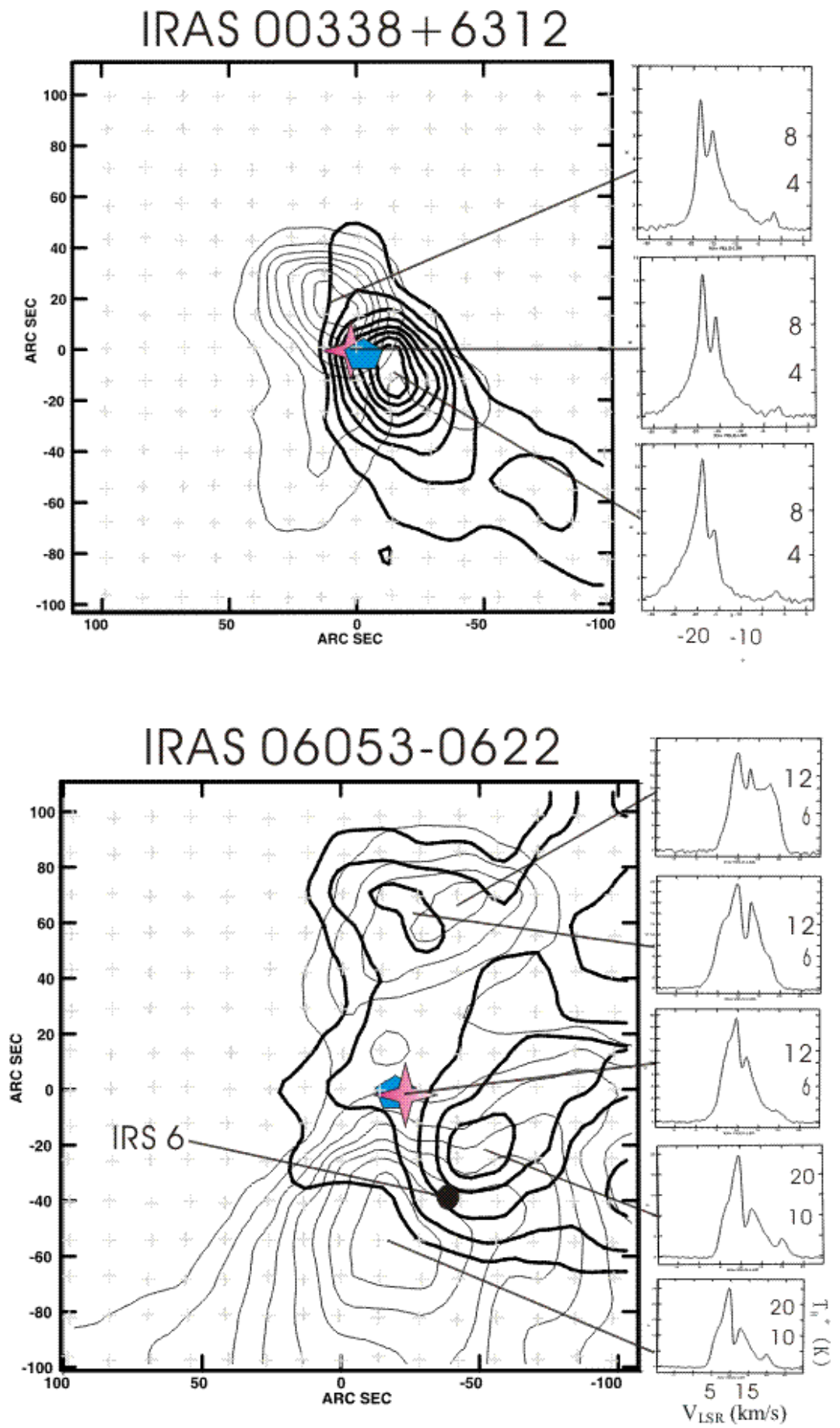

Fig. 1.- Maps of the integrated wing emission. The $6.7 \mathrm{GHz}$ Methanol maser emission is at the origin $(0,0)$. Red-shifted emission is indicated by thin contours, while blue-shifted emission by thick contours. Contour levels are 20 to $90 \%$ by steps of $10 \%$ of the peak integrated wing intensity. The star and pentagons symbols denote the IRAS source and HII region, respectively. Small crosses are the observed points. The spectra are plotted from the positions of the IRAS source, blue and red peaks, respectively. 

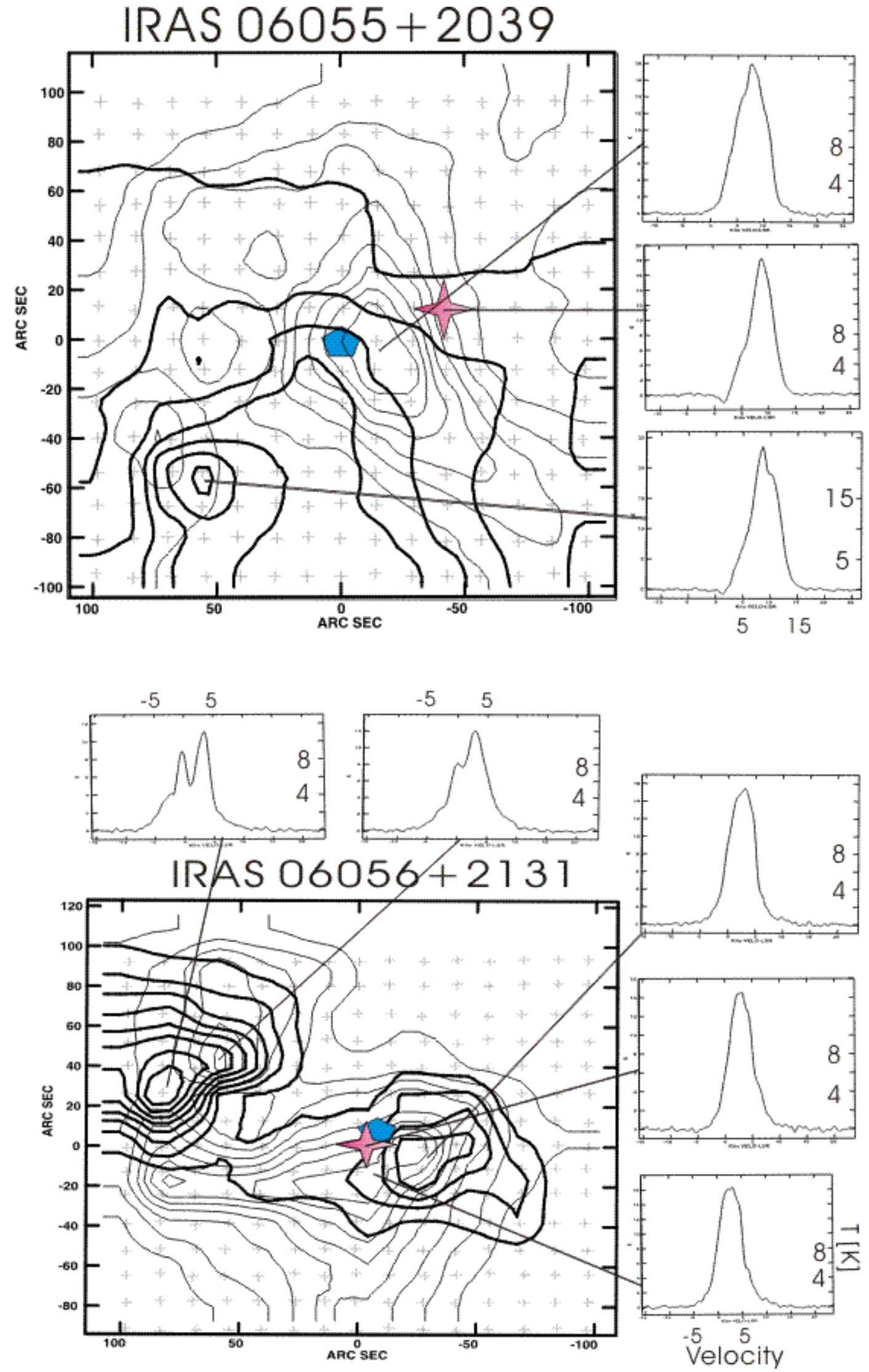

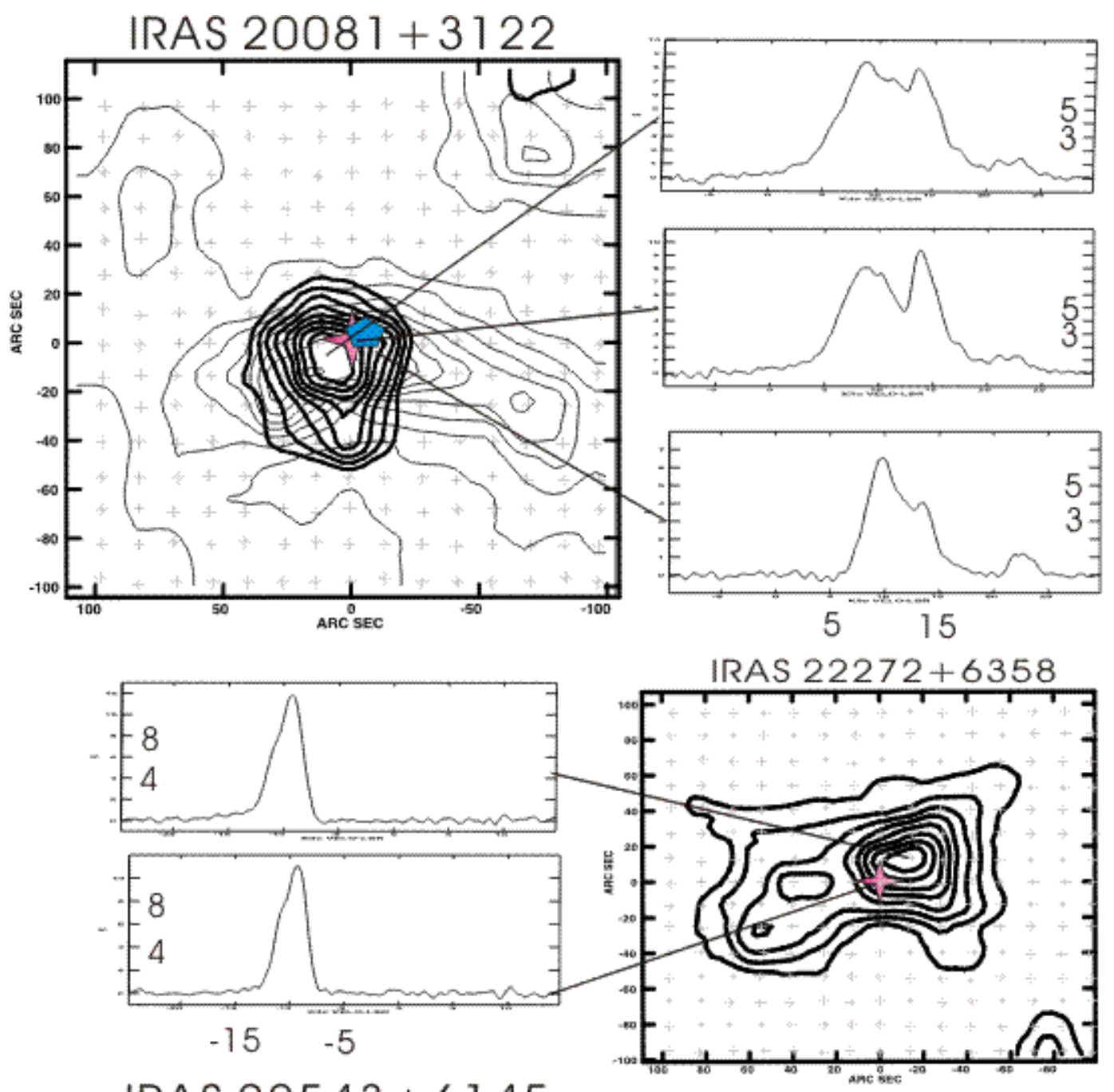

IRAS $22543+6145$

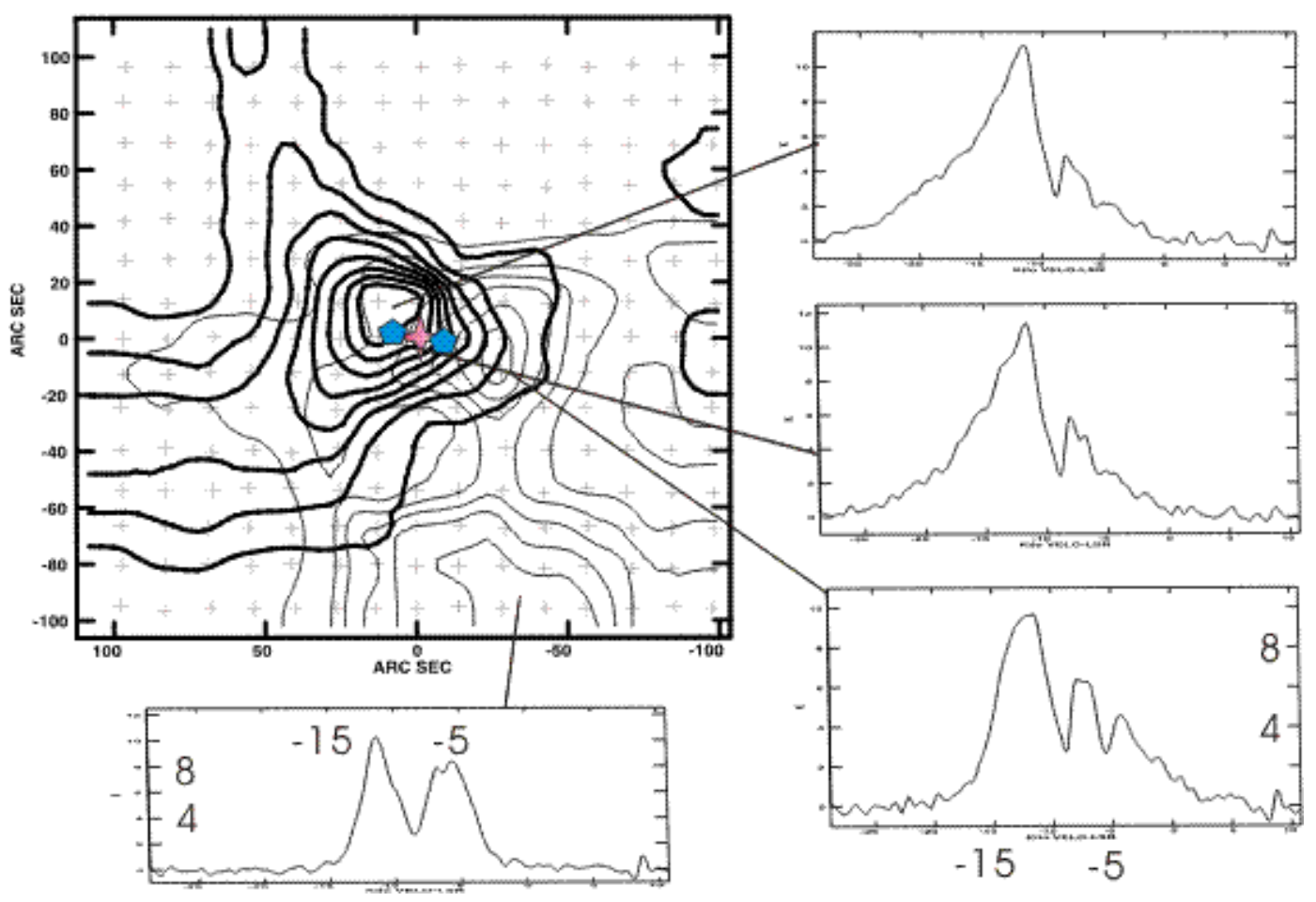

